\title{
Shining light on advanced NSCLC in 2017: combining immune checkpoint inhibitors
}

\author{
Meng Qiao, Tao Jiang, Caicun Zhou \\ Department of Medical Oncology, Shanghai Pulmonary Hospital \& Thoracic Cancer Institute, Tongji University School of Medicine, Shanghai \\ 200433, China \\ Contributions: (I) Conception and design: C Zhou; (II) Administrative support: None; (III) Provision of study materials or patients: None; (IV) \\ Collection and assembly of data: M Qiao, T Jiang; (V) Data analysis and interpretation: None; (VI) Manuscript writing: All authors; (VII) Final \\ approval of manuscript: All authors. \\ Correspondence to: Caicun Zhou. Department of Medical Oncology, Shanghai Pulmonary Hospital \& Thoracic Cancer Institute, Tongji University \\ School of Medicine, No. 507, Zhengmin Road, Shanghai 200433, China. Email: caicunzhoudr@163.com.
}

\begin{abstract}
The treatment landscape has changed since the immune checkpoint inhibitors were approved in the treatment of non-small cell lung cancer (NSCLC). Although the promising clinical benefit from programmed death-1/programmed death ligand-1 (PD-1/PD-L1) inhibitors was observed in the second or subsequent line treatment of patients who progressed on chemotherapy, it has a long way for single PD-1/ PD-L1 inhibitor to move forward to the frontline without a predictive biomarker. Tumor response is far from satisfactory without selection and primary or acquired resistance to PD-1/PD-L1 inhibitors hampered their utility. Therefore, it is crucial to determine a strategy that can optimize the application of immune checkpoint inhibitors and increase the numbers of the responders. Multiple combination approaches based on PD-1/PD-L1 inhibitors are designed and aimed to boost anti-tumor response and benefit a broader population. In this review, we will integrate the updated clinical data to highlight the four most promising combination strategies in advance NSCLC: combination of checkpoint inhibition with chemotherapy, antiangiogenesis, immunotherapy and radiotherapy. We further discuss the issues needed to be addressed and perspectives in the context of "combination era".
\end{abstract}

Keywords: Non-small cell lung cancer (NSCLC); combination strategy; programmed death-1 (PD-1); programmed death ligand-1 (PD-L1); immune checkpoint inhibitor

Submitted Jan 16, 2018. Accepted for publication Apr 11, 2018.

doi: $10.21037 /$ jtd.2018.04.99

View this article at: http://dx.doi.org/10.21037/jtd.2018.04.99

\section{Introduction}

Recent success in cancer immunotherapy has revolutionized the treatment landscape for patients with advanced nonsmall cell lung cancer (NSCLC), especially the rapid development of immune checkpoint inhibitors. There are three programmed death-1/programmed death ligand-1 (PD-1/PD-L1) inhibitors monotherapy approved in the treatment of NSCLC since 2015, namely nivolumab (PD-1 inhibitor), pembrolizumab (PD-1 inhibitor) and atezolizumab (PD-L1 inhibitor). At present, all of the three have been approved for second-line or subsequent line treatment of advanced NSCLC for their promising antitumor effect compared with standard chemotherapy (1-4). However, only pembrolizumab was approved for the firstline therapy for those patients with PD-L1 expression $\geq 50 \%$ and long-lasting response rate only achieves in $20-45 \%$ highly molecularly selected patients $(5,6)$. Besides, there are several limitations of using PD-L1 expression as a predictive biomarker for clinical use. Firstly, PD-L1 is not an absolute biomarker to predict the response to PD-1/PD-L1 inhibitors since tumor response could be observed in PD-L1 negative patients and even patients with high PD-L1 expression could be non-responders 
$(7,8)$. Secondly, focal PD-L1 expression will be inaccurately interpreted in small specimens, such as needle biopsies. Moreover, owing to the tumor heterogeneity, PD-L1 expression in multiple lesions from individual patient and multiple sites in one tumor lesion varies over time (9). Finally, the timing of biopsy collection influences the PDL1 status and before application of immune checkpoint inhibitors, other therapies may alter the PD-L1 expression. There is no standardized assay for PD-L1 testing and acknowledged cut-off to define the positive PD-L1 expression (10-12). In the context of the lack of biomarker to predict the response to single immune checkpoint inhibitor and the primary resistance and the emergence of the acquired resistance in initial responders to PD-1/PDL1 blockade (13), it is urgent to focus on rationalizing and broadening the utility of immune checkpoint inhibitors. The idea about combination based on PD-1/PD-L1 inhibitors comes up then $(14,15)$.

According to statistics, there are 1,105 combination clinical trials that combine anti-PD-1/PD-L1 agents with other therapies. Among them, anti-CTLA-4 agents and chemotherapies are the two most prevalent classes of therapies being combined with anti-PD-1/PD-L1 agents, with 251 and 170 studies, respectively. Radiotherapies and anti-VEGFA agents are also top prevalent partners as well, with 64 and 43 studies, respectively (16). In this current review, we will mainly discuss these four most promising and prevalent combinations in the treatment of advanced NSCLC: combination of checkpoint inhibition with chemotherapy, anti-angiogenesis, immunotherapy and radiotherapy. Finally, we will further discuss the matters needed to be concerned and future directions on the combination strategies.

\section{Combination of checkpoint inhibition with chemotherapy}

Chemotherapy has been the standard first-line treatment strategy for advanced NSCLC patients without molecular selections for decades. Most chemotherapeutic agents are considered to be immuno-suppressants and triggered the cytotoxic death of the cancer cells all this time, however, accumulating data suggested that the success of conventional chemotherapeutics is depending on the ability to stimulate the anti-tumor immune response (17). The immunological mechanisms behind the application of chemotherapy, including induction of immunogenic cell death (ICD) (18), the elimination of immunosuppressive cells (MDSCs,
T-regulatory cell) (19), upregulation of MHCI and PD-L1 expression on tumor cells $(20,21)$, enhancement of crosspresentation of tumor antigens, and better penetration of $T$ cells into the tumor (22), prompt the attempt to combine the conventional chemotherapy with $\mathrm{PD}-1 / \mathrm{PD}-\mathrm{L} 1$ inhibitors $(17,23)$. In addition, pemetrexed, which is a normal chemotherapeutic agent applied in the treatment of non-squamous NSCLC has been reported that it exerts positive effects on the intra-tumor $\mathrm{T}$ cell-mediated immune response independently of platinum agents and hence, enhances the effects of PD-L1 antibody in mouse tumor model (24).

Early results presented in a randomized, phase I/II clinical trial, KEYNOTE-021 cohort A-C demonstrated that pembrolizumab in combination with different chemotherapy regimens, including carboplatin/paclitaxel (cohort A, with any histology), carboplatin/paclitaxel + bevacizumab (cohort $\mathrm{B}$, with non-squamous histology) and carboplatin/pemetrexed (cohort C, with non-squamous histology) were well-tolerated and yielded promising antitumor efficacy regardless of PD-L1 status and dose of pembrolizumab 2 or $10 \mathrm{mg} / \mathrm{kg}$ (25). Particularly, $71 \%$ of patients $(17 / 24)$ in cohort $C$ achieved overall response. Therefore, based on the substantial clinical activity, KEYNOTE-021 cohort $\mathrm{G}$ was performed to further explore the synergy of pembrolizumab plus carboplatin/pemetrexed. According to the published data in 2016 (26), 123 patients were enrolled to receive either concurrent combination strategy with 24 months of pembrolizumab and indefinite pemetrexed maintenance therapy or chemotherapy alone followed by indefinite pemetrexed maintenance therapy. The incidence of grade 3 or worse treatmentrelated adverse effects was higher in the pembrolizumab + chemotherapy arm $(39 \%$ in combination arm vs. $26 \%$ in control arm) and the adverse events were manageable. Moreover, the higher ORR (55\% vs. 29\%) and significant clinical benefit were observed in combination arm with longer PFS (13.0 vs. 8.9 months). This promising strategy thus prompted FDA to grant an accelerated approval to pembrolizumab in combination of pemetrexed/ carboplatin as a first-line treatment for advanced nonsquamous NSCLC, regardless of PD-L1 expression. In 2017, updated data from KEYNOTE-021 G was reported with 18.7 months of median follow-up. Fifty-seven percent patients in pembrolizumab + chemotherapy achieved overall response, whereas, $32 \%$ in the chemotherapy group. In the subgroup analysis, regardless of PD-L1 expression, patients in combination arm had higher ORR 
rate. Particularly, $62 \%$ of patients with $\mathrm{PD}-\mathrm{L} 1$ expression less than $1 \%$ achieved overall response in combination arm whereas $17 \%$ of the same level of PD-L1 expression achieved the overall response in chemotherapy arm. PFS was significantly improved in combination arm (19.0 vs. 8.9 months, HR $=0.54, \mathrm{P}=0.0067)$. Patients in the combination arm got OS benefit as well with longer followup statistics (NR vs. 20.9 months, $\mathrm{HR}=0.59, \mathrm{P}=0.0344$ ) (27). However, this promising combination has not been approved by European Medicines Agency (EMA), possibly because it was the phase II results and needed further evaluation in larger population. Hence, two phase III trials, namely KEYNOTE-189 (NCT02578680) and KEYNOTE-407 (NCT02775435) which compared pembrolizumab + platinum/pemetrexed in non-squamous population and pembrolizumab + carboplatin-paclitaxel/ nab-paclitaxel in squamous population, respectively are ongoing and mature data are warranted.

In terms of nivolumab, the preliminary clinical benefit from a phase I study, CheckMate 012 was firstly reported in 2016 (28). 56 patients received nivolumab plus standard chemotherapy concurrently and 4 different regimens were set: nivolumab $10 \mathrm{mg} / \mathrm{kg}+$ gemcitabine/cisplatin (for squamous histology), nivolumab $10 \mathrm{mg} / \mathrm{kg}+$ pemetrexed/ cisplatin (for adenocarcinoma histology), nivolumab $10 \mathrm{mg} / \mathrm{kg}+\mathrm{paclitaxel} / \mathrm{carboplatin}$ (for any histology) and nivolumab $5 \mathrm{mg} / \mathrm{kg}+$ paclitaxel/carboplatin (for any histology). Encouraging response was achieved regardless of PD-L1 expression with ORR ranged from 33\% to $47 \%$ and 24 weeks PFS ranged from $51 \%$ to $71 \%$. Of note, 2 -year OS rate $(62 \%)$ was highest in the nivolumab plus paclitaxel/carboplatin group. After that, 3-year updated survival data reported in 2017 WCLC showed that for all patients, ORR has achieved $46 \%$ with 6 months of median PFS and 19.2 months of median OS. ORR and OS were similar in patients with positive PD-L1 expression or not $(<1 \%$ vs. $>1 \%)$. The 3 -year OS rate of $25 \%$ in the enrolled patients. This prolonged survival data supported the further exploration in this combination as first-line treatment of advanced NSCLC regardless of PD-L1 expression and CheckMate 227 (NCT02477826) which is an ongoing randomized, phase III study.

GP28328 (NCT01633970), was a phase Ib study designed to assess the safety, pharmacology and preliminary efficacy of atezolizumab administered in combination with bevacizumab and/or with chemotherapy in multiple types of solid tumors. Arm C, D, E (Arm C: atezolizumab + carboplatin/paclitaxel; Arm D: atezolizumab + carboplatin/ pemetrexed; Arm E: atezolizumab + carboplatin/nabpaclitaxel) were set for NSCLC patients with no prior chemotherapy. Updated to August 2016, 76 patients were enrolled and evaluated. Patients who were enrolled in Arm D achieved the highest ORR (64\%) with most significant clinical benefit. However, this result needs to be carefully interpreted for small numbers of participants (25 patients) and wide $95 \%$ CI. Several phase III trials are ongoing (Table 1).

\section{Combination of checkpoint inhibition with angiogenesis inhibitors}

VEGF, a proangiogenic molecule plays a key role in the process of angiogenesis, however, it also acts as a key mediator in a development of immunosuppressive microenvironment (29,30). Given that the immunosuppressive property of VEGF, it is evident that the anti-angiogenesis agents not only normalize the vascular formation but also stimulate the immune response $(31,32)$. Our previous study showed that anti-angiogenesis agent could eliminate the immune suppressive immune cells, including Tregs and MDSCs in lung cancer murine model which were consistent with results from other types of tumor $(33,34)$, however, the number of those suppressive immune cells will increase after a transient decrease which means that, single antiangiogenic agent is not supposed to produce the durable anti-tumor effect (unpublished data). To achieve the maximum anti-tumor response and considering the reciprocal effect between VEGF/VEGFR pathway and immune system, combining anti-angiogenesis and immune checkpoint inhibitors is possible to re-educate the tumor microenvironment from the suppressive status to an activated status.

A preclinical study from our team reported in 2016 WCLC showed that the combination of low-dose apatinib $(60 \mathrm{mg} / \mathrm{kg})$ and PD-L1 antibody demonstrated most significant anti-tumor effect by eliminating the number of suppressive immune cells and increasing the infiltration of $\mathrm{CD}^{+} \mathrm{T}$ cells (unpublished data). This promising preclinical result provided the rationale to combine these two blockades together and built a solid theory foundation for an ongoing clinical trial carried out in our center, combination of SHR-1210, a PD-1 antibody and apatinib in the treatment of advanced NSCLC. The preliminary result will be reported in 2018 ASCO.

Recently, the late-breaking results from a phase III trial, IMpower 150 reported in 2017 ESMO Immuno Oncology 


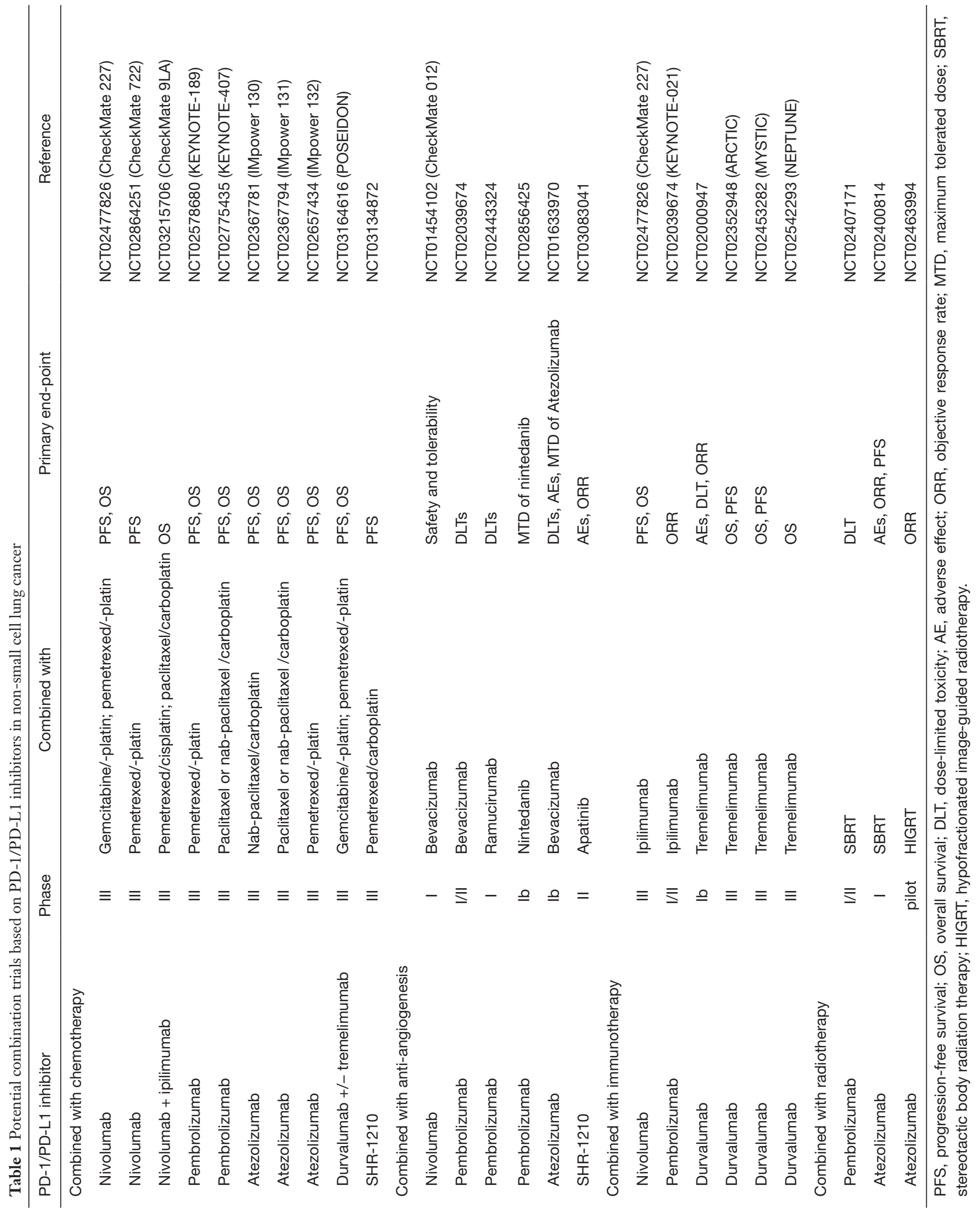


Congress confirmed the synergistic effect through a triple-therapy, namely atezolizumab, bevacizumab and chemotherapy. A total of 1,202 patients were enrolled and three Arms were designed in IMPOWER 150 (Arm A: atezolizumab + carboplatin/paclitaxel; Arm B: atezolizumab + bevacizumab + carboplatin/paclitaxel; Arm C: bevacizumab + carboplatin/paclitaxel). The results showed that compared with patients who received chemotherapy plus bevacizumab, patients who received additional atezolizumab without any genetic aberrations had longer PFS (6.8 vs. 8.3 months, $\mathrm{HR}=0.62, \mathrm{P}<0.001$ ) and higher 12 -month PFS rate (18\% vs. $37 \%)$. In the subgroup analysis, when the study population was broadened to include those with EGFR mutations and ALK rearrangements, the PFS benefit could be still observed $(\mathrm{HR}=0.61,95 \% \mathrm{CI}, 0.52-0.72)$. The combination delineated a safety profile without significantly increasing the incidence of serious AEs (Arm B vs. Arm C: $25.4 \%$ vs. $19.3 \%$ ). IMPOWER 150 is the first positive phase III combination trial applied in the first-line that showed the immuno-oncology strategy could reduce the risk of the disease getting worse in an unselected population with advanced non-squamous NSCLC.

The investigation of combining PD-1/PD-L1 inhibitor and anti-angiogenesis agent in the context of maintenance therapy seems dismal (35). Rizvi et al. reported that PFS data from nivolumab monotherapy (16 weeks in squamous and 21.4 weeks in non-squamous) or nivolumab plus bevacizumab group (37.1 weeks) were similar with other standard agents approved as maintenance therapy after chemotherapy. The exploration in another combination, ramucirumab, a VEGFR2 inhibitor and pembrolizumab in the treatment of NSCLC is still ongoing. The preliminary safety results from the dose-limiting toxicity (DLT) were reported in 2016 ASCO. Only three patients were evaluated in the cohort of NSCLC and no DLTs were observed. Therefore, 25 NSCLC patients were enrolled for the expansion of the phase Ib study. Eighty percent of patients had a decrease in target lesion and only one patient experienced grade 3 or 4 AEs. We are looking forward to more mature data about this combination in the treatment of NSCLC in a larger population (36).

\section{Combination of checkpoint inhibition with immunotherapies}

The rationale for combining of two immune checkpoint inhibitors, PD-L1/PD-1 inhibitors and CTLA-4 blockade is that two inhibitors act at different steps of the immune cycle, CTLA-4 at the initial phase of T-cell activation and PD-1/PD-L1 inhibitors inhibit the activation of effector T cell (37-39). Block the CTLA-4 pathway act in lymphoid compartment to expand the number and repertoire of tumor-reactive T cells (40) and block the PD-1/PD-L1 pathway act in the tumor microenvironment to re-activate the suppressive effector T cells. Additionally, gene analysis in vivo revealed that CTLA-4 blockade induces the proliferative signature predominantly in subset of transitional memory $\mathrm{T}$ cells and PD-1/PD-L1 inhibitors produce the gene changes in cytolysis and natural killer cell function (41). Thus, this complementary and non-overlapping mechanisms support the rationale and highlight the potential synergic anti-tumor effect when combining them together.

Clinical data regarding the assessment of safety and activity of combination nivolumab plus ipilimumab as firstline therapy was initially reported in 2016 (42). Seventyeight patients were enrolled to either receive nivolumab $3 \mathrm{mg} / \mathrm{kg}$ every 3 weeks ( $\mathrm{q} 3 \mathrm{w}$ ) plus ipilimumab $1 \mathrm{mg} / \mathrm{kg}$ every 12 weeks (q12w) or the same dose of nivolumab but ipilimumab $1 \mathrm{mg} / \mathrm{kg}$ every 6 weeks (q6w). Confirmed ORR was $47 \%$ and $48 \%$ in $\mathrm{q} 12 \mathrm{w}$ group and $\mathrm{q} 6 \mathrm{w}$ group, respectively. Median PFS was longer in the group of nivolumab plus ipilimumab ( $12 \mathrm{w})$ in comparison with nivolumab plus ipilimumab (q6w) [8.1 (5.6-13.6) vs. 3.9 (2.6-13.2) months]. This combination had a tolerable safety profile without any treatment-related deaths occurring. Additionally, when compared with nivolumab monotherapy, combination resulted in higher ORR (42\% vs. $23 \%$ ), longer PFS (8 vs. 3.6 months) and numerically higher 1 -year OS rate (76\% vs. $73 \%)$ (43). In the updated data reported in 2017 ASCO, promising results were shown in the subgroup analysis stratified by PD-L1 expression. The higher PD-L1 expression, the higher 1-year OS rate and ORR. Of note, patients who had 50\% PD-L1 expression or higher, 1 -year OS was $100 \%$ and $92 \%$ of them achieved overall response. Activity was observed in patients with $<1 \%$ PD-L1 expression. More investigations on the survival outcome with longer follow-up are participated. Additionally, this combination has been investigated cross multiple circumstances in the treatment of lung cancer, for instance, as neoadjuvant attempt in patients with early stage of lung cancer or patients with diagnosis of small cell lung cancer (SCLC).

For those who have high-risk of recurrence NSCLC patients after surgery, adjuvant chemotherapy is a standard 
regimen, however, only $5 \%$ OS benefit at 5 years. This unsatisfying data prompts to explore the role of neoadjuvant setting in IB-IIIA NSCLC patients. Higher tumor mutation burden and abundant tumor antigens are presented before the surgery which has been identified as the potential predictive biomarker for PD-1/PD-L1 inhibitors. Preliminary results from an ongoing study which aimed to explore the role of nivolumab in the neoadjuvant setting demonstrated the effective induction of pathological response without delaying the surgery. Additionally, combination of nivolumab and ipilimumab demonstrated promising antitumor activity in advanced NSCLC. Therefore, it is feasible to evaluate this combination in the neoadjuvant setting and we are looking for the data from CheckMate 816 (NCT02998528). This combination also showed promising anti-tumor activity with durable responses and manageable safety profiles in the treatment of SCLC (44) with $26 \%$ of 2 -year OS updated in the 2017 ASCO. Hence, nivolumab-ipilimumab combination regimen has been incorporated into NCCN guidelines for SCLC as second line treatment recommendation. Taken together, nivolumab-ipilimumab is the most promising combination so far, and hopefully, it can optimize the firstline strategy in the treatment of advanced NSCLC in the future.

There are several investigations on another combination of PD-L1 inhibitor and CTLA-4 blockade, termed durvalumab and tremelimumab. A phase I doseescalation study demonstrated that durvalumab $20 \mathrm{mg} / \mathrm{kg}$ every 4 weeks ( $\mathrm{q} 4 \mathrm{w}$ ) plus tremelimumab $1 \mathrm{mg} / \mathrm{kg}$ exhibited tolerated profile and regardless of PD-L1 expression, different dose combinations have shown anti-tumor activity (45). However, the phase III study on this combination (MYSTIC, NCT02453282) did not meet the primary endpoint of PFS compared to chemotherapy but this combination will continue to assess whether the combination extends the OS compared with standard of care (SoC) (46) (https://www.astrazeneca.com/). Two more phase III clinical trials regarding this combination are ongoing and mainly focusing on its effect on those patients with negative PD-L1 expression (NEPTUNE: NCT02542293; ARCTIC: NCT02352948) $(47,48)$ (Table 1).

The results reported in 2016 ASCO from KEYNOTE-021 cohort D and $\mathrm{H}$ concerning combination of pembrolizumab and ipilimumab as second-line therapy in NSCLC seems dismal with 7 months of follow-up. Compared with pembrolizumab alone, this combination increased the incidence of treatment-related AEs $(67 \%$ of patients suffered) without increasing the ORR (24\%). No link was observed between PD-L1 status and outcome. Longer follow-up is warranted to help define whether this combination merits further exploration (49).

\section{Combination of checkpoint inhibition with radiotherapies}

Radiotherapy plays a vital role in management of advanced NSCLC. It is widely known that radiotherapy not only exerts cytotoxic effects on tumor cells, but also alter the tumor microenvironment (50). The interaction between radiotherapy and immune response is complicated and multifactorial (51), and can be possibly concluded as followings: (I) enhances the expression of MHC-I on tumor cells (52); (II) produces more danger-associated molecular patterns (DAMPs) for cross-presentation (53); (III) activates the antigen-presenting cells (APCs); (IV) elicits the differentiation of M1 macrophages (54). Moreover, abscopal effect during the radiotherapy is defined as the response occurred in the tumors distant from the radiation site in the clinical setting. This phenomenon can be partly explained as systemic immune response which is triggered by the radiotherapy (55). However, the incidence of abscopal effect is comparatively low in radiotherapy alone but increases when combing with immunotherapy. Early in 2012, a clinical case reported the abscopal effect in a patient with melanoma treated with ipilimumab and radiotherapy (56). Besides, in preclinical models, combining PD-1 blockade and radiotherapy also induced abscopal effect $(57,58)$. It also demonstrated that PD-1 expression restrains the abscopal effect and a growing body of evidence have suggested that the PD-L1 expression is upregulated after radiation treatment $(59,60)$.

Our previous study demonstrated that combined conventionally fractionated radiotherapy and PD-L1 inhibitor synergistically enhance the anti-tumor immunity via eliminating the local accumulation of suppressive immune cells, such as Tregs and MDSCs and stimulating the infiltration of $\mathrm{CD}^{+} \mathrm{T}$ cells in NSCLC (61), and hence, resulting in a highly inflamed tumor. This synergistic effect was also observed in many types of cancer $(62,63)$.

Preclinical evidence suggested that proper dose and high quality of radiotherapy is acting as an in-situ vaccination that boosts immune response. However, the optimal sequence, the appropriate population and the clinical strategy to deal with the increasing risk of toxicity remain unclear.

Currently, multiple clinical trials are in design and 
ongoing in NSCLC, evaluating the combination of PD-1/PD-L1 inhibitors and radiotherapy, but to date, the data are not yet available (Table 1).

\section{Issues and perspectives}

\section{Issues-is combination a perfect choice?}

\section{Toxicity}

Accumulating evidence has proved that monotherapy with PD-1/PD-L1 inhibitor is well-tolerated, however, the risk of immune-related adverse effects increases with combination strategies owing to the emergence of immune cascades. In the published data from CheckMate 021 (28), 95\% of patients who received the nivolumab plus chemotherapy had any grade of adverse effects and $45 \%$ of them reported grade 3 or worse treatment-related AEs. However, in CheckMate 026 (64), $71 \%$ patients in nivolumab group occurred any grade of AEs whereas, grade 3 or worse AEs were only observed in $18 \%$ patients. Similar data was also observed in updated safety data from KEYNOTE-021-G (27), $93 \%$ of patients had any grade of events with treatment of pembrolizumab plus chemotherapy whereas $76.6 \%$ of patients with treatment of pembrolizumab alone occurred AEs according to the updated analysis of KEYNOTE-024 (65). Nivolumab plus ipilimumab $(\mathrm{q} 12 \mathrm{w})$ or $(\mathrm{q} 6 \mathrm{w})$ also doubled the risk incidence of grade 3-4 treatment related AEs compared with nivolumab monotherapy (42\% vs. 31\% vs. 19\%) (43). It is not surprising to observe such data on safety profile in combination exploration based on the immune checkpoint inhibitors and some trials were suspended for the high incidence of toxicity. For instance, TATTON, an exploratory trial with the aim of combining osimertinib and durvalumab in the treatment of EGFR-mutant NSCLC (66). However, the interstitial lung disease was incredibly high, with $38 \%$ patients suffered. No doubt, immune checkpoint inhibitors are systemic therapy and immune-related adverse-effects could not be avoided. Combing two systemic therapies possibly lead to the clinical benefit but also the unexpected toxicity which could decrease the life quality of the patients who had already suffered from the advanced cancer and pain. In a word, toxicity is the major concern and hurdle in front of us. The toxicities are not simply imagined and different from traditional chemotherapy. When caught early, the toxicities can be managed, however, if severe or identified late and managed not properly, the toxicities could be life-threatening, such as pneumonitis, colitis, nephritis, etc.
Is combination a good choice? Is combination a right path to cure cancer? Good or bad, hard to say.

\section{Cost-effectiveness}

There is no doubt that several months extension of PFS or OS is the substantial progress in the treatment of lung cancer. However, before this particular treatment strategy is applied in the real world, there is an integrated factor including three critical elements (clinical benefit, toxicity, and cost) that needs to be evaluated, termed 'value'. 'Value' is defined as a measure of outcomes achieved per monetary expenditure (67). As the rapid development of medicine, we aim to deliver the high-quality and affordable cancer care (68). Despite scientists and oncologists have tried their best on the way to design a perfect strategy in the treatment of lung cancer with comparatively high efficacy and safety profile, as for patients, they consider not only efficacy, but also the quality of life and financial burden (69). From their perspectives, they won't choose a regimen with high out-of-pocket costs but only extend life by only weeks or months or not at all (70). Additionally, combination therapies based on the immune checkpoint inhibitors not only lead to the improvement in the anti-tumor efficacy, but also the increased toxicity and unprepared financial distress. Whether the out-of-pocket costs on combination strategies is proportionally to extend the lifetime needs to be addressed. Hence, ASCO Board has developed a framework which put the patients at the center to forge a broad consensus on how to define the value of a cancer treatment-comparing the relative clinical benefit, toxicity, and cost of treatment in the medical oncology setting. By using the specific calculation methods, three parts of the scores are included and finally, the Net Health Benefit (NHB) score is presented to comprehensively demonstrate the value of the regimen compared to the SoC (71). Taken pembrolizumab as monotherapy (KEYNOTE-024) and as combination with chemotherapy (KEYNOTE-021-G) versus chemotherapy alone as an example $(5,27)$. According to the updated ASCO value framework (72), the combination regimen got +41 points $/ 130$ points in NHB whereas the cost of this regimen was 2.5 times (618,889 USD) of chemotherapy and the pembrolizumab as monotherapy got +51 points $/ 130$ points in NHB whereas the cost was 1.7 times $(368,917$ USD) of chemotherapy. This NHB score illustrated the comprehensive value of a potential strategy and assisted in decision making in the clinical scenarios. We hope that, in the future, the framework will allow the patients to decide the weight of 
each elements according to their own preferences to achieve the real "personalized" treatment strategy.

\section{Perspectives}

The optimal sequence and the timing for each possible combination are crucial and the outcome of the combination might be dismal if dose of each agent is wrongly applied. For instance, it has been shown that lower dose of antiangiogenic agents instead of higher dose have superior immune-stimulating effects when combined with immunotherapy (73). In terms of combination of radiotherapy and anti-PD-L1 blockade, Dovedi et al. pointed out that concurrent radiation and anti-PD-L1 treatment, but not sequential treatment, resulted in durable tumor control and were well-tolerated by patients (74). However, the ideal fraction or dose of radiation that combined with immunotherapy to exert better effects is still not determined. The success of a combination relies on the proper timing, dose and mode which should be investigated by adequate and solid preclinical support. Additionally, extrapolation of radiation dose from animal to human studies is not straightforward (75). Interpretation of the preclinical results should be based on the deep understanding of tumor biology.

Although PD-L1 expression has been paid most attention (5), not all people with inflamed immune profile, high PD-L1 expressing status are responsive to PD-1/ PD-L1 inhibitors. Tumor-infiltrating lymphocytes, tumor mutational burden (TMB), immune gene signature including those encoding interferon $\gamma(I F N G)$, granzyme $\mathrm{A}$ and $\mathrm{B}(G Z M A$ and $G Z M B)$, and perforin 1 (PFR1), etc., are developing to become new biomarkers (76-78). Single biomarker seems weak and unreliable to predictive the response rate to immune checkpoint inhibitors, while when used in combination with PD-L1 expression, the predictive power might be enhanced. It is urgent to create a biomarker-based model to guide the selection of individuals who are most likely to respond to PD-1/PD-L1 inhibitors as single agent or combination regimens, particularly, which combination is the most appropriate for individual patient. It requires that we should have deeper understanding of tumor immune microenvironment and the key step of limiting the normal operation of immune cycle in each immune status.

Reasonable interpretation from the clinical trials and the intermediate end points are the underpinnings for the development of potential immuno-combinations. Stephen J. Gould, who was diagnosed in 1982 with abdominal mesothelioma. He was informed after the surgery that this type of cancer is incurable with median mortality of 8 months after discovery. Three years later, in his famous essay, 'The median isn't the message', he firstly mentioned that it was necessary to distinguish the medians from milestones since the medians could diminish the hope of the individual patient (79). Although the medians could capture the clinical benefit offered by a particular therapy, such as EGFR-TKIs (80), however, it also bias us to ignore the efficacy of a class of drug which produced a delayed effect and long-term benefits, such as immunotherapy. The advantage of the immunotherapy is often missed in the median outcomes and only manifests when examining the tail of the survival curve. Therefore, focus on the tail of the curve and determine the milestone in the analysis of survival curve in the clinical trials that included the immunotherapy as an arm are of vital importance. In a meta-analysis performed by Blumenthal et al, there was an association between the milestone rate of OS at 12 months and OS HR $\left(\mathrm{R}^{2}=0.80,95 \%\right.$ CI, 0.63-0.91), but lack of association with other parameters, such as PFS milestone ratio at 9 months and ORR milestone ratio at 6 months (81). It is not appropriate to use the milestone rate as the primary end-point in the pivotal study. However, the milestone rate should be incorporated into secondary end-point in long-term follow up, particular for those trials comparing two immuno-combinations or versus immunotherapy alone. For instance, KEYNOTE-021-G also reported the 18-month OS rates in both combination and control group (70\% vs. 56\%) (27). Besides, different combinations that have integrated the immune checkpoint inhibitors may demonstrate the unique pattern of response, progression and survival curve, hence, it is urgent to define the optimal milestones in each combination setting to identify the early signal of the anti-tumor activity and guide for decision-making in whether continuing or not (82).

Research on immunotherapy is not supposed to limit to the immune checkpoint inhibitors and combination based on the immune checkpoint inhibitors. Investigation on more forms of immunotherapy is required. Chimeric antigen receptor (CAR) T-cell therapy is a special and effective form of adoptive cell transfer (ACT). T cells are obtained from patients and after genetically modified, those $\mathrm{T}$ cells express the CAR that directly and specifically target the tumor-associated antigen, such as CD19 in patients with leukemia or lymphoma $(83,84)$. CAR-T cell therapy has demonstrated remarkable success in hematologic malignancies and FDA has approved the first CAR-T 
product in the treatment of acute lymphoid leukemia (ALL). Despite favorable responses of CAR-T in patients with non-solid malignancies, the outcome in solid tumor is far from satisfactory partly owing to the lack of unique antigens, antigen loss in cancer cells, the suppressive tumor microenvironment and modified $\mathrm{T}$ cells are rarely accumulating in tumors $(85,86)$. Many investigations are ongoing on converting this gloomy option in solid tumor. A previous study illustrated that combination of $\mathrm{PD}-1$ inhibitor and anti-HER2 CAR-T cells enhanced the anti-tumor effect in breast carcinoma mouse model (87). However, given the high cost of CAR-T and PD-1/PD$\mathrm{L} 1$ inhibitor, Li et al. reported that they generated an antiPD-1 self-secreting CAR. $\alpha$ PD-1-T cells model which are efficient in tumor eradication (88). Moreover, Milner et al. recently found that tissue-resident memory $\mathrm{T}$ cell $\left(\mathrm{T}_{\mathrm{RM}}\right)$ and circulating memory $\mathrm{T}$ cell $\left(\mathrm{T}_{\mathrm{CM}}\right)$ population have different gene expression and chromatin accessibility and the transcription factor, Runx 3 is a key regulator of $T_{R M}$ cell differentiation and homeostasis. High expression of Runx3 in $\mathrm{T}_{\mathrm{RM}}$ enhanced tumor-specific $\mathrm{CD} 8^{+} \mathrm{T}$ cell abundance and promotes residency. This finding prompts us that Runx 3 might be an ideal partner in modification of circulating $\mathrm{T}$ cells extracted from the patients (89). Taken together, no absolute gain or in vain. As much deeper understanding of immune system and tumor biology, there is always a solution to overcome the barriers. Hopefully, CAR-T could act as the leading role in the treatment of NSCLC in the upcoming decades.

Immune responses against cancer are highly heterogeneous, indicating that individualized immunotherapy should be employed, based on the immune status of the individual patient. Chen and Mellman (90) have analyzed that there are three basic immune profiles in the TME, namely immuneinflamed phenotype, immune-excluded phenotype and immune-desert phenotype. The latter two phenotypes can be classified as uninflamed phenotypes. Therefore, to make the personalized immunotherapy strategy, the first step is to evaluate the immune profiles in patients before receiving the therapy. For those with inflamed immune phenotypes ('hot' tumors), anti-tumor immune response was arrested in tumor microenvironment, (I) maximize the functions of T cells: combined with immunostimulatory agents, such as IL-2, CSF-1R, etc.; (II) unleash the immune-block in the TME, such as combined with other immune checkpoint inhibitors, CTLA-4 blockade or antagonists to co-inhibitory molecules, such as TIGIT, LAG-3, TIM-3, etc. For those with uninflamed immune phenotypes ('cold' tumors), the strategy should be made based on the following aspects: (I) promote the peripheral $\mathrm{T}$ cells infiltrate or penetrate to tumor sites: combined with anti-angiogenic agents or CAR-T cell therapy; (II) recruit more effector T cells: combined with agonists to stimulatory molecules, such as OX-40, CD137, CD40, etc. For those with lack of identified immune profiles, combined with chemotherapy might work owing to chemotherapeutic agents enhance immune response in multiple steps of cancer-immunity cycle (91).

\section{Concluding remarks}

It is the best of the times for the progress of immunooncology ever since. With the purpose of benefiting broader population without restriction of PD-L1 expression, combination strategy is the certain trend of further investigation. The promising results from KEYNOTE021-G and IMPOWER 150 reported in 2017 shined the light on the advanced NSCLC, however, in the upcoming 2018, we urge more discussion to define optimal biomarkers to guide future personalized immunotherapy and the clinical trials that take aim at raising the tail of the survival curve. Dickens once said in the 'A Tale of Two Cities', We had everytbing before us, we had notbing before us. Hopefully, with the deeper understanding of complicated immune system, our ultimate cancer goal-'cure' can be achieved in the near future.

\section{Acknowledgements}

We thank Dr. Antonio Rossi and Dr. Silvia Novello to invite us to make contribution on this topic.

Funding: This study was supported in part by grants from the National Science Foundation of China (No. 81672286), Key Discipline of Shanghai Municipal Commission of Health and Family Planning (No. 2017ZZ02012), Major Basic Research Program of Shanghai Municipal Science and Technology Commission (No. 16JC1405900), Leading Program of Shanghai Municipal Science and Technology Commission (No. 16411964400).

\section{Footnote}

Conflicts of Interest: The authors have no conflicts of interest to declare.

\section{References}

1. Herbst RS, Baas P, Kim DW, et al. Pembrolizumab 
versus docetaxel for previously treated, PD-L1-positive, advanced non-small-cell lung cancer (KEYNOTE-010): a randomised controlled trial. Lancet 2016;387:1540-50.

2. Rittmeyer A, Barlesi F, Waterkamp D, et al. Atezolizumab versus docetaxel in patients with previously treated non-small-cell lung cancer (OAK): a phase 3, openlabel, multicentre randomised controlled trial. Lancet 2017;389:255-65.

3. Brahmer J, Reckamp KL, Baas P, et al. Nivolumab versus Docetaxel in Advanced Squamous-Cell Non-Small-Cell Lung Cancer. N Engl J Med 2015;373:123-35.

4. Borghaei H, Paz-Ares L, Horn L, et al. Nivolumab versus Docetaxel in Advanced Nonsquamous Non-Small-Cell Lung Cancer. N Engl J Med 2015;373:1627-39.

5. Reck M, Rodriguez-Abreu D, Robinson AG, et al. Pembrolizumab versus Chemotherapy for PD-L1Positive Non-Small-Cell Lung Cancer. N Engl J Med 2016;375:1823-33.

6. Attili I, Passaro A, Pavan A, et al. Combination immunotherapy strategies in advanced non-small cell lung cancer (NSCLC): Does biological rationale meet clinical needs? Crit Rev Oncol Hematol 2017;119:30-9.

7. Aguiar PN, Jr., Santoro IL, Tadokoro H, et al. A pooled analysis of nivolumab for the treatment of advanced non-small-cell lung cancer and the role of PD-L1 as a predictive biomarker. Immunotherapy 2016;8:1011-9.

8. Lipson EJ, Forde PM, Hammers HJ, et al. Antagonists of PD-1 and PD-L1 in Cancer Treatment. Semin Oncol 2015;42:587-600.

9. Jamal-Hanjani M, Wilson GA, McGranahan N, et al. Tracking the Evolution of Non-Small-Cell Lung Cancer. N Engl J Med 2017;376:2109-21.

10. Smith J, Robida MD, Acosta K, et al. Quantitative and qualitative characterization of Two PD-L1 clones: SP263 and E1L3N. Diagn Pathol 2016;11:44.

11. Takada K, Toyokawa G, Okamoto T, et al. A Comprehensive Analysis of Programmed Cell Death Ligand-1 Expression With the Clone SP142 Antibody in Non-Small-Cell Lung Cancer Patients. Clin Lung Cancer 2017;18:572-82.e1.

12. Hirsch FR, McElhinny A, Stanforth D, et al. PDL1 Immunohistochemistry Assays for Lung Cancer: Results from Phase 1 of the Blueprint PD-L1 IHC Assay Comparison Project. J Thorac Oncol 2017;12:208-22.

13. Syn NL, Teng MWL, Mok TSK, et al. De-novo and acquired resistance to immune checkpoint targeting. Lancet Oncol 2017;18:e731-41.

14. Sznol M. Blockade of the B7-H1/PD-1 pathway as a basis for combination anticancer therapy. Cancer J 2014;20:290-5.

15. Gotwals P, Cameron S, Cipolletta D, et al. Prospects for combining targeted and conventional cancer therapy with immunotherapy. Nat Rev Cancer 2017;17:286-301.

16. Tang J, Shalabi A, Hubbard-Lucey VM. Comprehensive analysis of the clinical immuno-oncology landscape. Ann Oncol 2018;29:84-91.

17. Galluzzi L, Zitvogel L, Kroemer G. Immunological Mechanisms Underneath the Efficacy of Cancer Therapy. Cancer Immunol Res 2016;4:895-902.

18. Garg AD, More S, Rufo N, et al. Trial watch: Immunogenic cell death induction by anticancer chemotherapeutics. Oncoimmunology 2017;6:e1386829.

19. Sevko A, Michels T, Vrohlings M, et al. Antitumor effect of paclitaxel is mediated by inhibition of myeloidderived suppressor cells and chronic inflammation in the spontaneous melanoma model. J Immunol 2013;190:2464-71.

20. Zhang P, Su DM, Liang M, et al. Chemopreventive agents induce programmed death-1-ligand 1 (PD-L1) surface expression in breast cancer cells and promote PD-L1mediated T cell apoptosis. Mol Immunol 2008;45:1470-6.

21. Peng J, Hamanishi J, Matsumura N, et al. Chemotherapy Induces Programmed Cell Death-Ligand 1 Overexpression via the Nuclear Factor-kappaB to Foster an Immunosuppressive Tumor Microenvironment in Ovarian Cancer. Cancer Res 2015;75:5034-45.

22. Zitvogel L, Galluzzi L, Smyth MJ, et al. Mechanism of action of conventional and targeted anticancer therapies: reinstating immunosurveillance. Immunity 2013;39:74-88.

23. Apetoh L, Ladoire S, Coukos G, et al. Combining immunotherapy and anticancer agents: the right path to achieve cancer cure? Ann Oncol 2015;26:1813-23.

24. Novosiadly R, Schaer D, Lu Z, et al. Pemetrexed Exerts Intratumor Immunomodulatory Effects and Enhances Efficacy of Immune Checkpoint Blockade in MC38 Syngeneic Mouse Tumor Model. J Thorac Oncol 2017;12:S2300.

25. Gadgeel SM, Stevenson J, Langer CJ, et al. Pembrolizumab (pembro) plus chemotherapy as front-line therapy for advanced NSCLC: KEYNOTE-021 cohorts A-C. J Clin Oncol 2016;34: abstr 9016.

26. Langer CJ, Gadgeel SM, Borghaei H, et al. Carboplatin and pemetrexed with or without pembrolizumab for advanced, non-squamous non-small-cell lung cancer: a randomised, phase 2 cohort of the open-label KEYNOTE-021 study. Lancet Oncol 2016;17:1497-508. 
27. Borghaei H, Langer C, Gadgeel S, et al. OA 17.01 Pemetrexed-Carboplatin Plus Pembrolizumab as FirstLine Therapy for Advanced Nonsquamous NSCLC: KEYNOTE-021 Cohort G Update. J Thorac Oncol 2017;12:S1791.

28. Rizvi NA, Hellmann MD, Brahmer JR, et al. Nivolumab in Combination With Platinum-Based Doublet Chemotherapy for First-Line Treatment of Advanced Non-Small-Cell Lung Cancer. J Clin Oncol 2016;34:2969-79.

29. Voron T, Colussi O, Marcheteau E, et al. VEGF-A modulates expression of inhibitory checkpoints on CD8+ T cells in tumors. J Exp Med 2015;212:139-48.

30. Ohm JE, Gabrilovich DI, Sempowski GD, et al. VEGF inhibits T-cell development and may contribute to tumorinduced immune suppression. Blood 2003;101:4878-86.

31. Manegold C, Dingemans AC, Gray JE, et al. The Potential of Combined Immunotherapy and Antiangiogenesis for the Synergistic Treatment of Advanced NSCLC. J Thorac Oncol 2017;12:194-207.

32. Huang Y, Goel S, Duda DG, et al. Vascular normalization as an emerging strategy to enhance cancer immunotherapy. Cancer Res 2013;73:2943-8.

33. Finke JH, Rini B, Ireland J, et al. Sunitinib reverses type-1 immune suppression and decreases T-regulatory cells in renal cell carcinoma patients. Clin Cancer Res 2008;14:6674-82.

34. Terme M, Pernot S, Marcheteau E, et al. VEGFAVEGFR pathway blockade inhibits tumor-induced regulatory $\mathrm{T}$-cell proliferation in colorectal cancer. Cancer Res 2013;73:539-49.

35. Rizvi NA, Antonia SJ, Shepherd FA, et al. Nivolumab (Anti-PD-1; BMS-936558, ONO-4538) Maintenance as Monotherapy or in Combination With Bevacizumab (BEV) for Non-Small Cell Lung Cancer (NSCLC) Previously Treated With Chemotherapy. Metastatic Non-Small Cell Lung Cancer. Int J Radiat Oncol Biol Phys 2014;90:S32.

36. Herbst RS, Bendell JC, Isambert N, et al. A phase 1 study of ramucirumab $(\mathrm{R})$ plus pembrolizumab $(\mathrm{P})$ in patients (pts) with advanced gastric or gastroesophageal junction (G/GEJ) adenocarcinoma, non-small cell lung cancer (NSCLC), or urothelial carcinoma (UC): Phase 1a results. J Clin Oncol 2016;34: abstr 3056.

37. Walunas TL, Lenschow DJ, Bakker CY, et al. CTLA-4 can function as a negative regulator of $\mathrm{T}$ cell activation. Immunity 1994;1:405-13.

38. Krummel MF, Allison JP. CD28 and CTLA-4 have opposing effects on the response of $\mathrm{T}$ cells to stimulation.
J Exp Med 1995;182:459-65.

39. Chambers CA, Kuhns MS, Egen JG, et al. CTLA-4mediated inhibition in regulation of $T$ cell responses: mechanisms and manipulation in tumor immunotherapy. Annu Rev Immunol 2001;19:565-94.

40. Kvistborg P, Philips D, Kelderman S, et al. Anti-CTLA-4 therapy broadens the melanoma-reactive CD8+ T cell response. Sci Transl Med 2014;6:254ra128.

41. Das R, Verma R, Sznol M, et al. Combination therapy with anti-CTLA-4 and anti-PD-1 leads to distinct immunologic changes in vivo. J Immunol 2015;194:950-9.

42. Hellmann MD, Rizvi NA, Goldman JW, et al. Nivolumab plus ipilimumab as first-line treatment for advanced nonsmall-cell lung cancer (CheckMate 012): results of an open-label, phase 1, multicohort study. Lancet Oncol 2017;18:31-41.

43. Gettinger S, Rizvi N, Chow L, et al. OA03.01 FirstLine Nivolumab Monotherapy and Nivolumab plus Ipilimumab in Patients with Advanced NSCLC: LongTerm Outcomes from CheckMate 012. J Thorac Oncol 2017:Abstract OA03.1.

44. Antonia SJ, Lopez-Martin JA, Bendell J, et al. Nivolumab alone and nivolumab plus ipilimumab in recurrent smallcell lung cancer (CheckMate 032): a multicentre, openlabel, phase 1/2 trial. Lancet Oncol 2016;17:883-95.

45. Antonia S, Goldberg SB, Balmanoukian A, et al. Safety and antitumour activity of durvalumab plus tremelimumab in non-small cell lung cancer: a multicentre, phase $1 \mathrm{~b}$ study. Lancet Oncol 2016;17:299-308.

46. Peters S, Antonia S, Goldberg SB, et al. 191TiP: MYSTIC: a global, phase 3 study of durvalumab (MEDI4736) plus tremelimumab combination therapy or durvalumab monotherapy versus platinum-based chemotherapy (CT) in the first-line treatment of patients (pts) with advanced stage IV NSCLC. J Thorac Oncol 2016;11:S139-40.

47. Mok T, Schmid P, Aren O, et al. 192TiP: NEPTUNE: A global, phase 3 study of durvalumab (MEDI4736) plus tremelimumab combination therapy versus standard of care (SoC) platinum-based chemotherapy in the first-line treatment of patients (pts) with advanced or metastatic NSCLC. J Thorac Oncol 2016;11:S140-1.

48. Planchard D, Yokoi T, McCleod MJ, et al. A Phase III Study of Durvalumab (MEDI4736) With or Without Tremelimumab for Previously Treated Patients With Advanced NSCLC: Rationale and Protocol Design of the ARCTIC Study. Clin Lung Cancer 2016;17:232-6.e1.

49. Gubens MA, Sequist LV, Stevenson J, et al. Phase I/II study of pembrolizumab (pembro) plus ipilimumab (ipi) as 
second-line therapy for NSCLC: KEYNOTE-021 cohorts D and H. J Clin Oncol 2016;34: abstract 9027.

50. Teng F, Kong L, Meng X, et al. Radiotherapy combined with immune checkpoint blockade immunotherapy: Achievements and challenges. Cancer Lett 2015;365:23-9.

51. Rodel F, Frey B, Gaipl U, et al. Modulation of inflammatory immune reactions by low-dose ionizing radiation: molecular mechanisms and clinical application. Curr Med Chem 2012;19:1741-50.

52. Reits EA, Hodge JW, Herberts CA, et al. Radiation modulates the peptide repertoire, enhances $\mathrm{MHC}$ class I expression, and induces successful antitumor immunotherapy. J Exp Med 2006;203:1259-71.

53. Sharma A, Bode B, Wenger RH, et al. gamma-Radiation promotes immunological recognition of cancer cells through increased expression of cancer-testis antigens in vitro and in vivo. PLoS One 2011;6:e28217.

54. Klug F, Prakash H, Huber PE, et al. Low-dose irradiation programs macrophage differentiation to an iNOS(+)/ M1 phenotype that orchestrates effective $T$ cell immunotherapy. Cancer Cell 2013;24:589-602.

55. Demaria $\mathrm{S}, \mathrm{Ng} \mathrm{B}$, Devitt $\mathrm{ML}$, et al. Ionizing radiation inhibition of distant untreated tumors (abscopal effect) is immune mediated. Int J Radiat Oncol Biol Phys 2004;58:862-70.

56. Postow MA, Callahan MK, Barker CA, et al. Immunologic correlates of the abscopal effect in a patient with melanoma. N Engl J Med 2012;366:925-31.

57. Sharabi A, Nirschl C, Ceccato T, et al. Role of Radiation Therapy in Inducing Antigen Specific Antitumor Immune Responses When Combined With Anti-PD1 Checkpoint Blockade: Mechanism and Clinical Implications. Int J Radiat Oncol Biol Phys 2014;90:S1.

58. Park SS, Dong H, Liu X, et al. PD-1 Restrains Radiotherapy-Induced Abscopal Effect. Cancer Immunol Res 2015;3:610-9.

59. Weichselbaum RR, Liang H, Deng L, et al. Radiotherapy and immunotherapy: a beneficial liaison? Nat Rev Clin Oncol 2017;14:365-79.

60. Deng L, Liang H, Burnette B, et al. Irradiation and antiPD-L1 treatment synergistically promote antitumor immunity in mice. J Clin Invest 2014;124:687-95.

61. Gong X, Li X, Jiang T, et al. Combined Radiotherapy and Anti-PD-L1 Antibody Synergistically Enhances Antitumor Effect in Non-Small Cell Lung Cancer. J Thorac Oncol 2017;12:1085-97.

62. Azad A, Yin Lim S, D'Costa Z, et al. PD-L1 blockade enhances response of pancreatic ductal adenocarcinoma to radiotherapy. EMBO Mol Med 2017;9:167-80.

63. Oweida A, Lennon S, Calame D, et al. Ionizing radiation sensitizes tumors to PD-L1 immune checkpoint blockade in orthotopic murine head and neck squamous cell carcinoma. Oncoimmunology 2017;6:e1356153.

64. Carbone DP, Reck M, Paz-Ares L, et al. First-Line Nivolumab in Stage IV or Recurrent Non-Small-Cell Lung Cancer. N Engl J Med 2017;376:2415-26.

65. Brahmer J, Rodriguez-Abreu D, Robinson A, et al. OA 17.06 Updated Analysis of KEYNOTE-024: Pembrolizumab vs Platinum-Based Chemotherapy for Advanced NSCLC With PD-L1 TPS $\geq 50 \%$. J Thorac Oncol 2017;12:S1793-4.

66. Ahn MJ, Yang J, Yu H, et al. 136O: Osimertinib combined with durvalumab in EGFR-mutant non-small cell lung cancer: Results from the TATTON phase Ib trial. J Thorac Oncol 2016;11:S115.

67. Feeley TW, Fly HS, Albright H, et al. A method for defining value in healthcare using cancer care as a model. J Healthc Manag 2010;5 5:399-411; discussion 411-2.

68. Shih YC, Ganz PA, Aberle D, et al. Delivering highquality and affordable care throughout the cancer care continuum. J Clin Oncol 2013;31:4151-7.

69. Zafar SY, Alexander SC, Weinfurt KP, et al. Decision making and quality of life in the treatment of cancer: a review. Support Care Cancer 2009; 17:117-27.

70. Weinstein MC. Should physicians be gatekeepers of medical resources? J Med Ethics 2001;27:268-74.

71. Schnipper LE, Davidson NE, Wollins DS, et al. American Society of Clinical Oncology Statement: A Conceptual Framework to Assess the Value of Cancer Treatment Options. J Clin Oncol 2015;33:2563-77.

72. Schnipper LE, Davidson NE, Wollins DS, et al. Updating the American Society of Clinical Oncology Value Framework: Revisions and Reflections in Response to Comments Received. J Clin Oncol 2016;34:2925-34.

73. Huang Y, Yuan J, Righi E, et al. Vascular normalizing doses of antiangiogenic treatment reprogram the immunosuppressive tumor microenvironment and enhance immunotherapy. Proc Natl Acad Sci U S A 2012;109:17561-6.

74. Dovedi SJ, Illidge TM. The antitumor immune response generated by fractionated radiation therapy may be limited by tumor cell adaptive resistance and can be circumvented by PD-L1 blockade. Oncoimmunology 2015;4:e1016709.

75. Monjazeb AM, Schoenfeld JD. Radiation dose and checkpoint blockade immunotherapy: unanswered questions. Lancet Oncol 2016;17:e3-4. 
76. Gibney GT, Weiner LM, Atkins MB. Predictive biomarkers for checkpoint inhibitor-based immunotherapy. Lancet Oncol 2016;17:e542-51.

77. Rizvi NA, Hellmann MD, Snyder A, et al. Mutational landscape determines sensitivity to PD-1 blockade in nonsmall cell lung cancer. Science 2015;348:124-8.

78. Ribas A, Robert C, Hodi FS, et al. Association of response to programmed death receptor 1 (PD-1) blockade with pembrolizumab (MK-3475) with an interferoninflammatory immune gene signature[abstract]. Polymer International 2015;37:141-7.

79. Gould SJ. The median isn't the message. Ceylon Med J 2004;49:139-40.

80. Fukuoka M, Wu YL, Thongprasert S, et al. Biomarker analyses and final overall survival results from a phase III, randomized, open-label, first-line study of gefitinib versus carboplatin/paclitaxel in clinically selected patients with advanced non-small-cell lung cancer in Asia (IPASS). J Clin Oncol 2011;29:2866-74.

81. Blumenthal GM, Zhang L, Zhang H, et al. Milestone Analyses of Immune Checkpoint Inhibitors, Targeted Therapy, and Conventional Therapy in Metastatic NonSmall Cell Lung Cancer Trials: A Meta-analysis. JAMA Oncol 2017;3:e171029.

82. Hellmann MD, Kris MG, Rudin CM. Medians and Milestones in Describing the Path to Cancer Cures: Telling "Tails". JAMA Oncol 2016;2:167-8.

83. Grupp SA, Kalos M, Barrett D, et al. Chimeric antigen receptor-modified $\mathrm{T}$ cells for acute lymphoid leukemia. $\mathrm{N}$

Cite this article as: Qiao M, Jiang T, Zhou C. Shining light on advanced NSCLC in 2017: combining immune checkpoint inhibitors. J Thorac Dis 2018;10(Suppl 13):S1534-S1546. doi: $10.21037 /$ jtd.2018.04.99
Engl J Med 2013;368:1509-18.

84. Park JH, Geyer MB, Brentjens RJ. CD19-targeted CAR T-cell therapeutics for hematologic malignancies: interpreting clinical outcomes to date. Blood 2016;127:3312-20.

85. Kiesgen S, Chicaybam L, Chintala NK, et al. Chimeric Antigen Receptor (CAR) T-Cell Therapy for Thoracic Malignancies. J Thorac Oncol 2018;13:16-26.

86. Kato D, Yaguchi T, Iwata T, et al. Prospects for personalized combination immunotherapy for solid tumors based on adoptive cell therapies and immune checkpoint blockade therapies. Nihon Rinsho Meneki Gakkai Kaishi 2017;40:68-77.

87. John LB, Devaud C, Duong CP, et al. Anti-PD-1 antibody therapy potently enhances the eradication of established tumors by gene-modified T cells. Clin Cancer Res 2013;19:5636-46.

88. Li S, Siriwon N, Zhang X, et al. Enhanced Cancer Immunotherapy by Chimeric Antigen Receptor-Modified T Cells Engineered to Secrete Checkpoint Inhibitors. Clin Cancer Res 2017;23:6982-92.

89. Milner JJ, Toma C, Yu B, et al. Runx3 programs CD8(+) $\mathrm{T}$ cell residency in non-lymphoid tissues and tumours. Nature 2017;552:253-7.

90. Chen DS, Mellman I. Elements of cancer immunity and the cancer-immune set point. Nature 2017;541:321-30.

91. Chen DS, Mellman I. Oncology meets immunology: the cancer-immunity cycle. Immunity 2013;39:1-10. 